\title{
Stem Cell Therapy: Are we Ready to use it?
}

\author{
Jia-Ming Chang*, Yi-Chien Lin and Pei-Yi Tsai
}

Department of Pharmacology, Institute for Drug Evaluation Platform, Development Center for Biotechnology, Taiwan, Republic of China

*Corresponding author: Department of Pharmacology, Institute for Drug Evaluation Platform, Development Center for Biotechnology, New Taipei City, Taiwan, Republic of China , Tel: +886 937074938; Email: jiaming@ntu.edu.tw

Rec date: February 25, 2014, Acc date: April 5 2014, Pub date: April 7, 2014

Copyright: (C) 2014 Chang JM, et al. This is an open-access article distributed under the terms of the Creative Commons Attribution License, which permits unrestricted use, distribution, and reproduction in any medium, provided the original author and source are credited.

\begin{abstract}
The regenerative medicine is a fast growing innovation of health care for replacement of damaged or dysfunctional human organs or tissues with functional normal. However, the shortage of graft donors is the major concern of this treatment. To solve this problem, pluripotent or multipotent stem cells, which have the potential to differentiate into multiple lineages, provide an unlimited source of desired cells for transplantation. Even though stem cell therapy became a promising tool for regenerative medicine, unfortunately some unexpected adverse effects occurred in such treatments. Especially the incidence of life-threatening event from malignant teratomas, which are generated from undifferentiated stem cells in graft, is greater than that from damaged organs or tissues per se. Here, in this article the safety issues associated with stem cell therapy are reviewed, and in warnings for patients who want to receive this therapy are also mentioned.
\end{abstract}

\section{Introduction}

During the past decades, many drugs have been developed for treating several diseases; these drugs modify cellular functions and defend against foreign microorganisms to restore normal conditions. However, if drugs cannot recover the loss of function occurring due to cell death, replacement of damaged tissues or dysfunctional organs with normal tissues provides a strategy to cure patients. Therapy with pluripotent or multipotent stem cells, which have the potential to differentiate in vitro to multiple lineages, should become the treatment of choice, as is the case with Haematopoietic Stem Cells (HSCs) therapy that is now used to treat several haematology-related diseases and fibroblasts that are being cultured on dressing materials to accelerate wound healing $[1,2]$. The first consideration is to increase the clinical benefits for patients, but physicians at the same time must also consider safety issues associated with using stem cells, particularly with regard to controlling cell proliferation and differentiation.

\section{Stem Cell Therapy}

Stem cell therapy is a medical treatment that introduces new pluripotent or multipotent stem cells into damaged tissue for curing disease or injury. It has been applied for treating numerous diseases, such as immune disorder, diabetes, neurodegenerative and heart diseases [3]. The first clinical case of stem cell therapy was conducted in late 1950s, which the bone marrow derived haematopoietic stem cells were used for treating the haematological disorders [4]. However, the first success comes out until late 1960s [5,6]. Currently, the stem cells used in clinics are mostly derived from the somatic one (tissuespecific stem cells), while only few derivate from embryonic stem cells are used in clinical trials. Among them, the intensive use of HSCs gives the best definition in blood-related disorders or in restoration of bone marrow after chemotherapy or radiotherapy. Besides, other somatic stem cells, such as mesenchymal, neural, adipose, and endothelial stem cells have also been reported in treating different diseases [2,3]. In this article, one case for immunological application with HSCs is discussed, in which the advantage or disadvantage by taking such therapy are mentioned for patients who are interested in stem cell therapy.

\section{Alternative Therapy for Multiple Sclerosis}

Multiple Sclerosis (MS) is a progressive autoimmune disease of the central nervous system in which nerve cells in the brain and spinal cord are damaged by immunity. Steroid for treating the acute phase inflammation of the brain is the inherent treatment. It is worth to note that long-term administration of steroids will cause severe side effects including psychosis, insomnia, headache, bone loss, suppression of the immune system, moon face, and stomach ulcers, and also increase the level of blood sugar. A strategy for MS treatment is the use of immune modulating agents, such as Avonex and Rebif. It has been reported that can attenuate the progression of physical disability and decrease the frequency of flare ups. Meanwhile, flu-like symptoms, depression, and a drop in blood cell and platelet number were occurred in such treatments [7]. Nevertheless, these therapies have only demonstrated relative risk reductions of approximately $30 \%$, and absolute risk reductions of 0.3 relapses per year in patients with multiple sclerosis [8]. Another choice for MS treatment is to use immunosuppressive agents alone or in combination with humanized monoclonal antibodies (natalizumab). The outcome of clinical studies demonstrated a $68 \%$ relative risk reduction (absolute risk reduction0.55 relapses per year) in annual relapse rate with natalizumab compared to placebo [9].Unfortunately, progressive multifocal leuko encephalopathy, which is an often fatal opportunistic infection caused by the JC virus, are reported in patients who receive natalizumab. Because of severe side effect, this drug was dropped from the market [10].

More recently, a potential therapy, so called AHSCT, is used for treating MS when patients fail to approved therapies. MS patients are transplanted with autologous Haematopoietic Stem-Cell (CD34+ HSCs) and immunity is shut down by intensive immune suppression prior to transplantation. The purpose of this therapy is first to destroy the auto reactive $\mathrm{T}$ cells where are located in the periphery and target 
organ by intensive immune suppression and the immunity is reconstituted further by transplantation of autologous stem cells $[11,12]$. It is believed that rebuilding of the immunity by means of AHSCT could reduce the hypersensitivity to autoantigens for a short period. Although obvious benefits in inflammatory parameters and disease progression were seen particularly in rapidly evolving severe multiple sclerosis, the risk of mortality, which dropped in the last years to $2-3 \%$, is still the main problem of this potential therapy [13].

\section{The Use of Embryonic Stem Cells}

Human Embryonic Stem Cells (hESCs) are totipotent cells that are derived from the inner cell mass of blastocyst-stage embryos. Totipotency property offers hESCs to differentiate into any required type of cells for different applications. Due to the use of embryo as source of hESCs, ethics considerations slow down the progress of embryonic stem cell therapy until 2009. In that year, the first clinical trial involving human embryonic stem cells had been approved by Food and Drug Administration (FDA) in the United States, which was sponsored by Geron Corporation. Promising results from their preclinical data demonstrated the therapeutic effect of using human Embryonic Stem Cell (hESC)- derived Oligodendrocyte Progenitor Cells(OPCs) to treat spinal cord injuries on a rat model [14]. Unexpectedly, the trial was terminated by business reasons on November 2011, and no formal result comes from the trial for publication. After that, other phase I/II clinical trials related to human embryonic stem cells sponsored by Advanced Cell Technology planned to use the hESC-derived retinal pigment epithelium cells for the treatment of Stargardt's Macular Dystrophy and Age-related Macular Degeneration. Even though the current statuses of each trial are still in recruiting, the outcome of first report from 2 patients was published in "The Lancet". In that report, outcome show an improved vision in patients without any concerns in safety issue of macular degeneration [15].

\section{Evaluation of Safety Issues Associated with Stem Cell Therapy}

Successful stem cell therapy for regenerative medicine is fraught with many hurdles. These include generating sufficient quantities of transplantable cells based on good manufacturing practices, following which the grafted cells must survive and integrate into surrounding tissue. Most importantly, the cells should appropriately function for the desirable period of time in the recipient.

Depending on the source of transplantable cells derived from either immunologically unmatched donor (allogeneic) or self-origin (autologous), different safety issues would be further concerned. For allogeneic transplantation, it generally encounters the chance of infection from grafted cells, and immune rejection may occur in the graft-versus-host disease. Suppression of rejection is needed and continued to treat patients, but unfortunately some unexpected risks will be evoked with long-term treatment such as infection and cancer occurrence. In spite of autologous transplantation preventing from these adverse effects, it still requires evaluation on cell viability, cell ability, and enough amounts to produce functional tissue.

To address safety issues from regular stem cell therapy, alternative way by using Induced Pluripotent Stem Cells (iPS) is proposed and considered. The iPS cells are generated from reprogramming of differentiated adult cells into pluripotent stage. In similar to embryonic stem cells, iPS cells have unlimited capacity in number with self-renew ability and it could differentiate into specific type of cells. According to these characteristics, iPS can regard as a stable source of therapeutic cell materials in stem cell therapy. Besides, reprogramming of somatic cells into iPS cells retained self-own histo compatibility could prevent from immune rejection. If mutations occurred in iPS cells, it can be repaired by replacement of functional gene, and those repaired cells can be further differentiated into intended cells before therapy. It is suggested that iPS cells therapy offers a promising way for providing personalized treatment to human diseases in the future $[16,17]$.

Even though iPS cells therapy has far-reaching potency in regenerative medicine, most studies in controlling their reprogramming and differentiation are still under development. It may be raised safety concerns in genetic and epigenetic abnormalities during processes [18]. Prior to clinical study, researchers must clarify deeply the molecular and cellular mechanisms in controlling of reprogramming and differentiation. Necessarily, the type of desired cells must be confirmed using genetic and biomarker analysis, followed by purification to the best possible extent. Thus far, the most predominant challenge of stem cell therapy has been to completely purify differentiated cells from undifferentiated ones. These undifferentiated cells have the potential of forming malignant teratomas, which is one of the worst outcomes in clinical treatment; however, the frequency of malignant teratoma formation is also influenced by many factors such as the site of injection, number of cells in the inoculum, addition of excipients, and inoculation with other cell populations $[19,20]$.

Beside the safety issue associated with undifferentiated cells, other potential risks of differentiated progenitor cells remain unknown. Current practice for monitoring the tumor-forming potential of each stem cell-derived transplant involves a xenograft animal model. However, the dose of cells administered to patients is much greater than that tested, and the long-term tumorigenic potential of clinical use may not be properly reflected in such preclinical models. The grafted cells seem to be more prone to generate tumors in the species from where it is derived [21].More understanding in development and pathogenesis of teratomas will provide us more important clues for safety assessment and physical examination in stem cell trial.

\section{Warnings about Stem Cell Therapy}

Although stem cells are promising for generating novel therapies for a variety of diseases and injuries, at present, the FDA has approved only one stem cell product, Hemacord, which is a human cord bloodderived product for use in allogeneic transplantation in case of certain blood cancers and inherited metabolic and immune-system disorders [22]. The use of stem cells still raises the prospect of several potential dangers. These safety issues must be addressed as a part of the riskbenefit ratio evaluation of stem cell-based biological products. The International Society for Stem Cell Research, an independent nonprofit stem cell research organization, has produced the "Patient Handbook on Stem Cell Therapies" to protect patients from stem cell therapies that have neither yet been proven to be safe nor effective [23]. Moreover, the handbook lists some major warning signs for patients who are considering stem cell therapy: (1) claims based on patient testimonials-patients may not have the expertise to carefully evaluate clinical research for understanding the true effects of the treatment and what to expect following the treatment, (2) multiple diseases treated with the same cells-different diseases would be expected to require equally different treatments, unless the diseases are 
related, (3) source of the cells and how the treatment will be performed may not be clearly documented patients should request clear documentation that includes detailed outlines of the medical practitioner's operating manual pertaining to the desired procedure and should ask the practitioner to clearly explain the treatment consent form, (4) claims that there are no associated risks there is always a risk involved with medical procedures, and (5) high cost of treatment or hidden costs-legitimate clinical trials should not charge patients, and patients should consider whether to pay for an unproven treatment.

\section{Conclusion}

For the successful development of stem cell-based therapy, clinicians need to develop novel technologies to improve the yield of differentiated cells, to exclude undifferentiated cells from differentiated ones via sorting or small-molecule compounds, and to generate in vivo cell imaging tools to track the location of the grafted cells. Moreover, preclinical research must gather information pertaining to toxicity, cell dosages, administration routes, and potential adverse effects. Previously unidentified safety issues should also be considered, including those that clinicians typically do not take into consideration. For example, "The methods used to expand the cells in culture might have contribution for tumor formation," according to Steven Goldman, an FDA advisory and chair of neurology at the University of Rochester Medical Center, New York [24]. Stem cell therapy seems like a promising approach to initiate a new era in curing diseases that could not have been cured using chemical drugs in the past. Finally, patient benefit should always be the first priority, and the decision to use or not to use stem cells should always be at the patient's discretion.

\section{References}

1. Ratcliffe E, Glen KE, Naing MW, Williams DJ (2013) Current status and perspectives on stem cell-based therapies undergoing clinical trials for regenerative medicine: case studies. Br Med Bull 108: 73-94.

2. Tabar V, Studer L (2014) Pluripotent stem cells in regenerative medicine: challenges and recent progress. Nat Rev Genet 15: 82-92.

3. Trounson A, Thakar RG, Lomax G, Gibbons D (2011) Clinical trials for stem cell therapies. BMC Med 9: 52.

4. THOMAS ED, LOCHTE HL Jr, LU WC, FERREBEE JW (1957) Intravenous infusion of bone marrow in patients receiving radiation and chemotherapy. N Engl J Med 257: 491-496.

5. Gatti RA, Meuwissen HJ, Allen HD, Hong R, Good RA (1968) Immunological reconstitution of sex-linked lymphopenic immunological deficiency. Lancet 2: 1366-1369.
6. Bach FH, Albertini RJ, Joo P, Anderson JL, Bortin MM (1968) Bonemarrow transplantation in a patient with the Wiskott-Aldrich syndrome. Lancet 2: 1364-1366.

7. Multiple Sclerosis: Causes, Symptoms, Treatments.

8. Rudick RA (2011) Multiple sclerosis, natalizumab, and PML: helping patients decide. Cleve Clin J Med 78 Suppl 2: S18-23.

9. Polman $\mathrm{CH}$, O'Connor PW, Havrdova E, Hutchinson M, Kappos L, et al. (2006) A randomized, placebo-controlled trial of natalizumab for relapsing multiple sclerosis. N Engl J Med 354: 899-910.

10. Baldwin KJ, Hogg JP (2013) Progressive multifocal leukoencephalopathy in patients with multiple sclerosis. Curr Opin Neurol 26: 318-323.

11. Mancardi G, Saccardi R (2008) Autologous haematopoietic stem-cell transplantation in multiple sclerosis. Lancet Neurol 7: 626-636.

12. Muraro PA, Douek DC, Packer A, Chung K, Guenaga FJ, et al. (2005) Thymic output generates a new and diverse TCR repertoire after autologous stem cell transplantation in multiple sclerosis patients. J Exp Med 201: 805-816.

13. Capello E, Vuolo L, Gualandi F, Van Lint MT, Roccatagliata L, et al. (2009) Autologous haematopoietic stem-cell transplantation in multiple sclerosis: benefits and risks. Neurol Sci 30 Suppl 2: S175-177.

14. Keirstead HS, Nistor G, Bernal G, Totoiu M, Cloutier F, et al. (2005) Human embryonic stem cell-derived oligodendrocyte progenitor cell transplants remyelinate and restore locomotion after spinal cord injury. J Neurosci 25: 4694-4705.

15. Schwartz SD, Hubschman JP, Heilwell G, Franco-Cardenas V, Pan CK, et al. (2012) Embryonic stem cell trials for macular degeneration: a preliminary report. Lancet 379: 713-720.

16. Wang, H (2011) Induced Pluripotent Stem Cells: To model, To Treat. J Cell Sci Ther 2: e103.

17. Jiang W, Zhang J, Yang X, Gao J (2012) Induced pluripotent stem cells:origins and directions. Human Genet Embryol S2: 002.

18. Pera MF (2011) Stem cells: The dark side of induced pluripotency. Nature 471: 46-47.

19. Amariglio N, Hirshberg A, Scheithauer BW, Cohen Y, Loewenthal R, et al. (2009) Donor-derived brain tumor following neural stem cell transplantation in an ataxia telangiectasia patient. PLoS Med 6: e1000029.

20. Cunningham JJ, Ulbright TM, Pera MF, Looijenga LH (2012) Lessons from human teratomas to guide development of safe stem cell therapies. Nat Biotechnol 30: 849-857.

21. Erdö F, Bührle C, Blunk J, Hoehn M, Xia Y, et al. (2003) Host-dependent tumorigenesis of embryonic stem cell transplantation in experimental stroke. J Cereb Blood Flow Metab 23: 780-785.

22. FDA approves first cord blood product (2011) Food and Drug Administration, HEMACORD.

23. ISSCR Patient Handbook on Stem Cell Therapies (2008).

24. Baker M (2009) Unregulated stem cell transplant causes tumours. Nature Reports stem cell. 